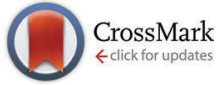

Cite this: J. Mater. Chem. C, 2015, 3, 8284

Received 3rd May 2015,

Accepted 28th June 2015

DOI: $10.1039 / c 5 t c 01234 a$

www.rsc.org/MaterialsC

\title{
Emerging strategies for the synthesis of monodisperse colloidal semiconductor quantum rods
}

\begin{abstract}
Guohua Jia, ${ }^{\star a}$ Shiqing $X u^{* b}$ and Aixiang Wang ${ }^{* c}$
Colloidal semiconductor quantum rods are exciting materials from both fundamental and technological points of view. The electronic and optical properties of these materials are governed by the decrease in the confinement of charge carriers along the long axis and by the cylindrical symmetry of the particles. Their intrinsic properties, such as highly controlled optical characteristics, are advantageous in biological labeling, photovoltaic devices and light emitting diodes. Thanks to the advances in the development of strategies for synthesizing colloidal semiconductor quantum rods that made these materials available. The availability of colloidal semiconductor quantum rods provides a platform for both the investigation of their stimulating properties and the exploration of their promising applications. This review article focuses on the recent advances in the strategies for the synthesis of monodisperse colloidal semiconductor quantum rods. The fundamental issues associated with the anisotropic growth of colloidal nanocrystals such as facet, surface energy, selective binding of the surfactant, growth kinetics and thermodynamics were addressed, interpreted and discussed. Synthetic strategies for colloidal semiconductor quantum rods, being different from one another, were introduced and the growth mechanisms were revealed. The progress on the synthetic strategies of colloidal semiconductor quantum rods provided the basis for many inspiring applications in the fields of photocatalysis, opto-electronic devices, bio-labeling and other advanced applications.
\end{abstract}

\section{Introduction}

Colloidal semiconductor quantum rods are at the forefront of nanoscience and nanotechnology. These nanocrystals manifest unique characteristics such as large absorption cross section, low lasing threshold, linearly polarized absorption and emission, and improved charge separation and transport compared with their counterparts such as spherical quantum dots. ${ }^{1-7}$ Furthermore, their intrinsic geometry opens many new opportunities for the assembly of elongated nanoparticles into organized superstructures and superlattices. ${ }^{8-14}$ All these desirable properties make colloidal semiconductor quantum rods promising candidates as building blocks in nanoscale materials and devices.

The availability of monodisperse colloidal semiconductor quantum rods with controlled aspect ratios is the prerequisite

\footnotetext{
${ }^{a}$ Nanochemistry Research Institute, Department of Chemistry, Curtin University, P.O. Box U1987, Perth, WA 6845, Australia. E-mail: guohua.jia@curtin.edu.au; Fax: +6189266 2300; Tel: +61892667882

${ }^{b}$ College of Materials Science and Engineering, China Jiliang University, Hangzhou 310018, China. E-mail: sxucjlu@163.com

${ }^{c}$ School of Chemistry and Chemical Engineering, Linyi University, Linyi 276005,

China. E-mail: wangaixiang1974@163.com
}

for their stimulating applications. This makes the development of synthetic strategies for these materials highly important and desirable. The significant advances in the synthesis of colloidal semiconductor quantum rods include the pioneering work on the preparation of colloidal semiconductor quantum rods by the nucleation of precursors and selective monomer attachment, ${ }^{15-17}$ which was subsequently modified by the dot/rod seeded rod growth approach. ${ }^{18-20}$ Other synthetic approaches for colloidal quantum rods include spherical particle coalescence by oriented attachment ${ }^{21-27}$ and catalyst-assisted growth. ${ }^{28-34}$ Most recently, the leading author of this Review and his colleagues had developed a general strategy for the synthesis of colloidal semiconductor quantum rods that led to the growth of monodisperse zinc chalcogenide quantum rods with controlled aspect ratios by a ripening process through thermodynamically driven material diffusion. ${ }^{9,35}$ All those advances on the synthetic strategies of colloidal semiconductor quantum rods addressed above made significant contributions to both the fundamental scientific knowledge and the application of colloidal semiconductor nanocrystals.

Current existing review articles provided significant insight on the fundamental properties of colloidal nanocrystals and their stimulating applications. However, those review articles 
either address a broad topic ${ }^{36-40}$ or do not include the up-todate advances in this research field..$^{41-45}$ This is the motivation of this review paper. Rather than a comprehensive review article of colloidal nanocrystals, this review specifically focused on the strategies that have been developed for synthesizing colloidal semiconductor quantum rods with controlled aspect ratios and good monodispersity. In Section 2, facets of both bulk and nanometre crystals will be introduced and discussed in order to understand the intrinsic properties of the facets such as surface energy, stability and growth speed. The discussion on the complicated case associated with colloidal nanocrystals, in which the surfactant and the binding energy between the surfactant and the facet play an important role, will also be included. Section 3 summarizes the existing and emerging synthetic strategies for the synthesis of semiconductor quantum rods and compares advantages and drawbacks associated with each synthetic strategy. At the end of this review article, challenges associated with the synthesis of colloidal semiconductor quantum rods will be included and prospects will be proposed to foresee some emerging synthetic strategies for the synthesis of colloidal anisotropic nanocrystals.

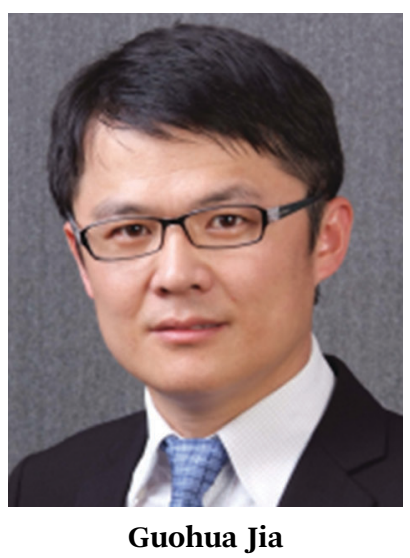

Guohua Jia received his $P h D$ in optical spectroscopy in 2009 from City University of Hong Kong under the supervision of Prof. Peter A. Tanner. From 2010 to 2014, he has been working with Professor Uri Banin as a postdoctoral fellow at the Hebrew University of Jerusalem in Israel, conducting his research on colloidal semiconductor nanocrystals. In January 2015, Dr Jia joined Curtin University as a Curtin Early Career Research Fellow.

His research focuses on the chemistry and physics of colloidal nanocrystals, with an emphasis on their application in optoelectronic devices, photocatalysis and bio-labeling.

\section{Facets of crystals: from bulk to nanoscale}

Bulk crystals grown from either water solution at mild temperatures or fluxes at high temperatures usually have unique morphologies manifested by the well-developed crystal facets. The development of crystal facets of a bulk crystal is supposed to be an intrinsic behaviour of the crystal and it is significantly dependent on the surface energy of each specific facet. Specifically, in the typical growth of a bulk crystal, the high-energy facet grows much faster than the low-energy facet and the fast growing facets will eventually disappear, leading to the formation of a bulk crystal terminated with low-energy facets. This is also true for colloidal nanocrystals, even the situation in this case becomes much more complicated due to the specifically preferable binding of organic surfactants on certain facets of nanocrystals compared with that of bulk crystals. ${ }^{25,46,47}$ Deep understanding the intrinsic properties of crystal facets and the functionalities of organic surfactants will give significant insights on anisotropic growth of colloidal nanocrystals and enrich the fundamental knowledge on how to synthesize elongated nanostructures with controlled aspect ratios and high monodispersity. Herein, a hexagonal wurtzite ZnO bulk crystal and a hexagonal wurtzite CdSe nanocrystal were specifically included, compared and interpreted. We chose them as two specific representative examples because of not only their crystallographic similarities but also their analogous optical and electronic properties. We will illustrate how anisotropic growth of crystals can be successfully controlled in terms of the surface energy of the facets in the bulk crystals and the binding energy between those facets and organic surfactant molecules in colloidal nanocrystals, as addressed in detail below.

ZnO has two main phases hexagonal wurtzite and cubic zinc blende, of which the wurtzite structure is most stable under ambient conditions and thus most common. The wurtzite structure of $\mathrm{ZnO}$ crystallizes into a noncentrosymmetric structure with a space group of $P 6_{3} m c$. In such a wurtzite structure, the anisotropic feature exists on both opposite sides of a basal plane wafer due to the different atomic arrangements at their surfaces, as shown in Fig. $1 \mathrm{a}^{48}$ There is a zinc-rich layer on the

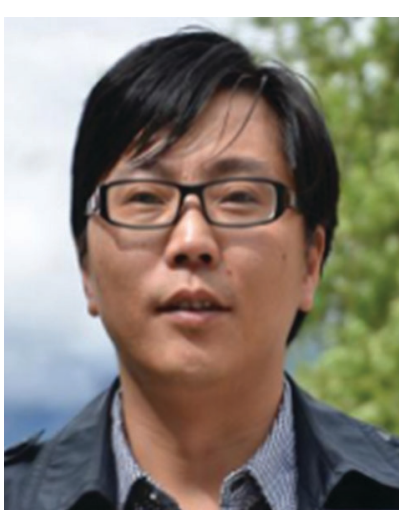

Shiqing Xu
Shiqing $\mathrm{Xu}$ received his $\mathrm{PhD}$ in 2005 from Shanghai Institute of Optics and Fine Mechanics, Chinese Academy of Sciences under the supervision of Prof. Zhonghong Jiang. $\mathrm{He}$ is a professor at China Jiliang University. His research focuses on the quantum dots, inorganic phosphors and glass ceramic nanocomposites, with an emphasis on their application in white light emitting diodes and sensors.

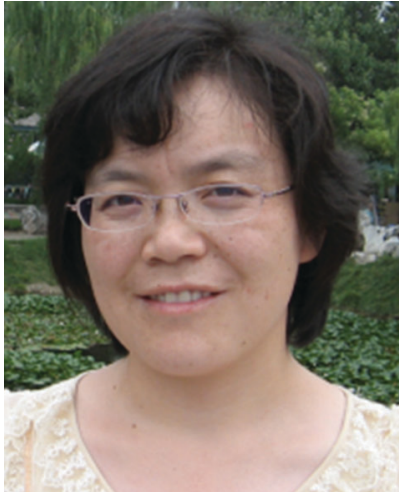

Aixiang Wang received her PhD in plasma synthesis in 2008 from Northwest Normal University under the supervision of Prof. Jinzhang Gao. Currently, she is an associate professor at Linyi University, conducting research on the synthesis, properties and application of quantum dotmolecularly imprinted polymer composite materials.

Aixiang Wang 
(a)

(b)
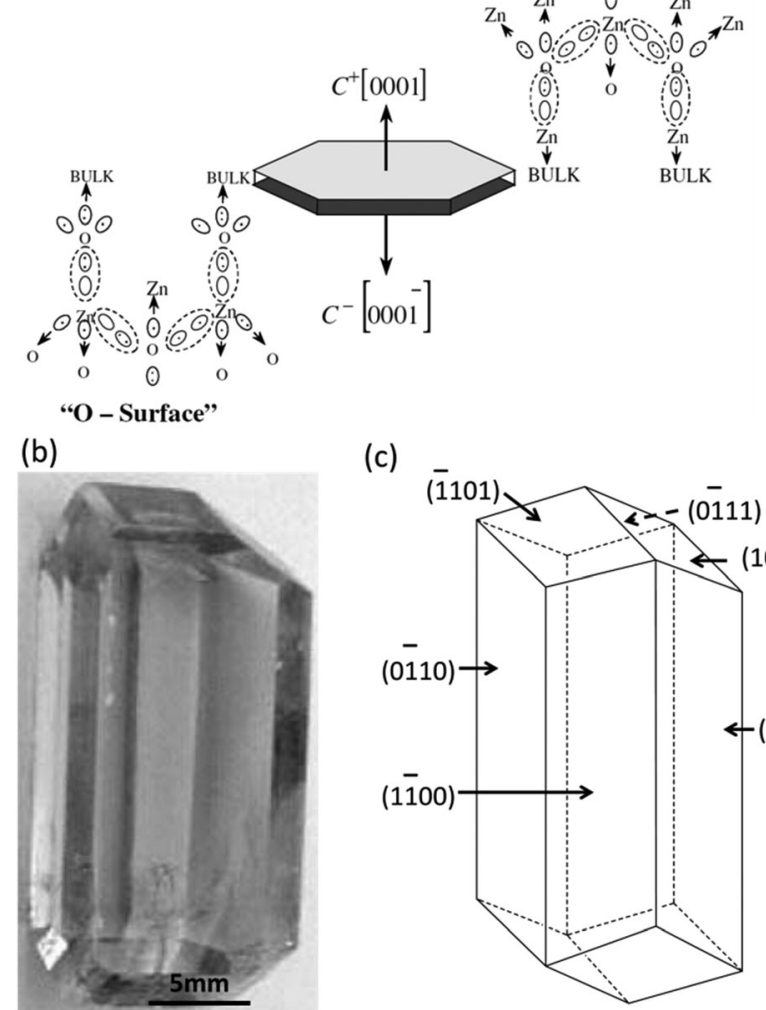

(c)

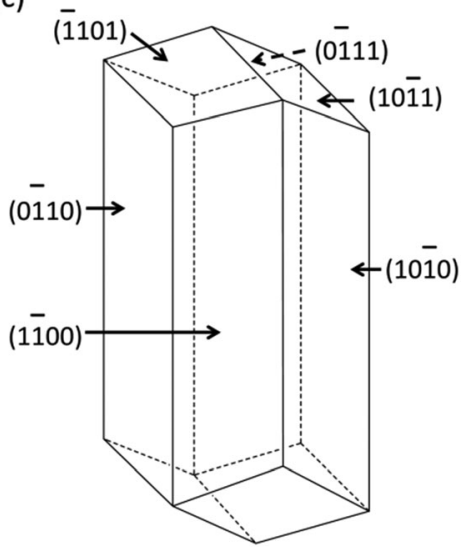

Fig. 1 Anisotropic growth of ZnO bulk crystals. (a) Electronic charge distribution of ZnO basal faces. (b) Photography of a ZnO bulk crystal grown by the hydrothermal method. (c) The model crystallite corresponding to (b). Panels ( $a$ ) and (b and c) reproduced with permission from ref. 48 and 49, with permission of Wiley-VCH and Elsevier, respectively.

$\mathrm{C}^{+}$side of the basal plane whereas the $\mathrm{C}^{-}$side consists of an oxygen-rich layer. This leads to uneven distribution of electric charge that causes significantly different growth rates of growth planes in the hydrothermal growth. ${ }^{48}$ Under ideal conditions, the growth rates of facets of a wurtzite $\mathrm{ZnO}$ crystal by the hydrothermal method show a descending order as $\nu(0001)>$ $\nu(01 \overline{1} \overline{1})>\nu(0 \overline{1} 10)>\nu(01 \overline{1} 1)>\nu(000 \overline{1})$.

A bulk ZnO single crystal with well-developed facets has been successfully grown by the hydrothermal method in an autoclave at mild temperatures (Fig. 1b). ${ }^{49}$ In a typical synthesis of $\mathrm{ZnO}$, a bulk ZnO crystal was grown from supercritical water at mild temperatures $\left(300-400{ }^{\circ} \mathrm{C}\right)$ and high pressure $(80-100 \mathrm{MPa})$. A mixture of alkali hydroxides containing $\mathrm{LiOH}$ and $\mathrm{KOH}$ was used as the solvent. The produced bulk ZnO crystal has an elongated rod feature along the $c$-axis with an aspect ratio of about 2.2, manifested by nine well-developed facets (Fig. 1b). Three pairs of facets parallel to the $c$-axis indexed as (1010), $(1 \overline{1} 00)$ and $(0 \overline{1} 10)$ are integrated into a hexagonal prism by

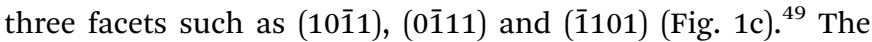
growth rate of the plane (0001) is much faster than that of the side facets and the fast growth rate produced the elongated anisotropic rod architecture with three pairs of parallel facets being the prominent facets that dominate the morphology of the bulk ZnO crystal.

As mentioned above in the case of bulk crystal growth, the most stable facets, which are supposed to have the lowest surface energy, dominate the final shape of the bulk crystal. Compared with the bulk ZnO crystal, wurtzite CdSe quantum rods also have well-developed facets, ${ }^{50,51}$ but the situation is much more complicated for CdS quantum rods since they are mainly grown in a surfactant-rich environment and have a large portion of surface atoms. Fig. 2a shows a schematic illustration of the anisotropic growth of CdSe quantum rods in a mixture of organic solutions of trioctylphosphine acid (TOP) and trioctylphosphine oxide (TOPO) in the presence of octadecylphosphonic acid (ODPA) and hexylphosphonic acid (HPA) as the surfactants. In analogy to a ZnO bulk crystal, each individual wurtzite CdS quantum rod also has three pairs of well-developed side facets indexed as (1100), (1010) and (10ī0). The (0001) end facet on one end of the quantum rod is Cd terminated, whereas the $(000 \overline{1})$ one on the other end is Se terminated. ${ }^{50,51}$ The end facets and side facets are

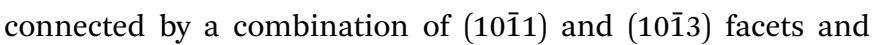
steps, forming the truncated anisotropic elongated rod architecture (Fig. 2c).
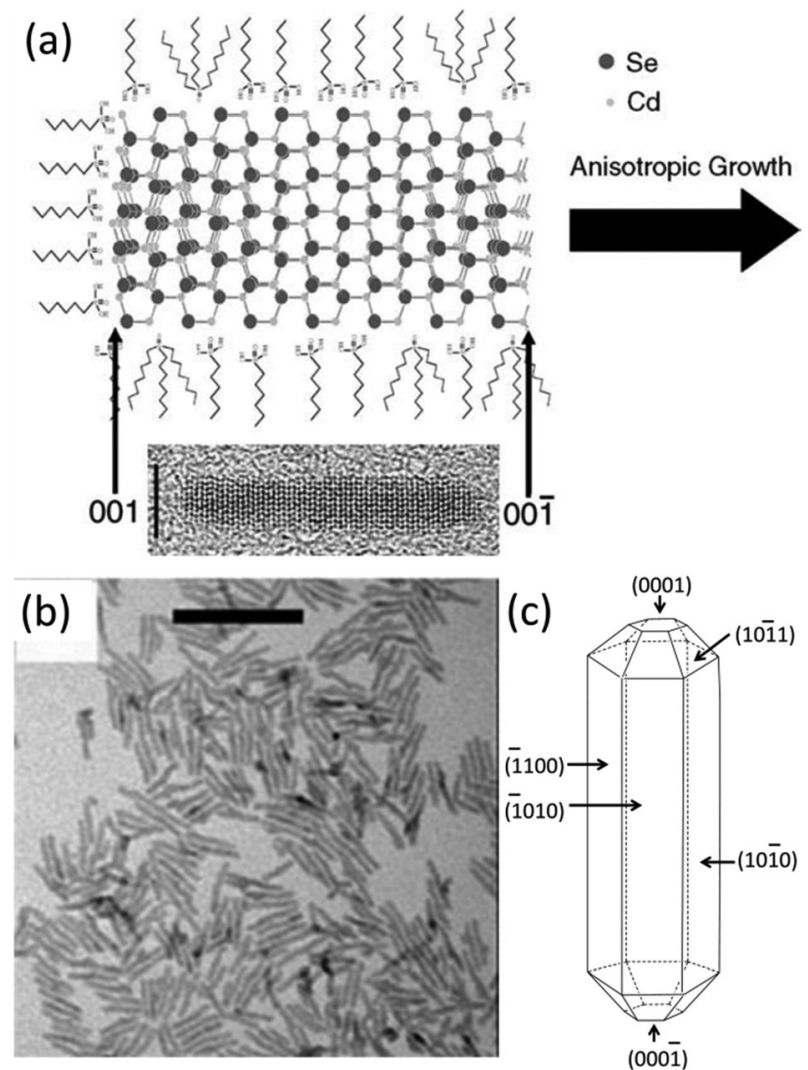

Fig. 2 Anisotropic growth of CdSe quantum rods. (a) Proposed growth mechanism for CdSe quantum rods. An HRTEM image of CdSe quantum rods aligned in the same direction as the scheme. (b) TEM image of CdSe quantum rods. (c) The model crystallites corresponding to (b). The model crystallites show the indices for various surface types. Panels (a), (b) and (c) reproduced with permission from ref. 50, 15 and 51, with permission of the Plenum Publishing Corporation, McMillan Magazines and the American Chemical Society, respectively. 
In a real growth environment, the colloidal nanocrystals are grown in a liquid solution rich of surfactants. The stability of each facet of a nanocrystal will not depend on the surface energy of each facet only. It will significantly be modified by the binding energy between the organic surfactant molecules and the facets of the nanocrystals. In the case of a cylindrical wurtzite CdSe nanocrystal, the surface energies of the side facets and end facets as well as the connecting facets and steps within the nanocrystal are expected to be closely associated with their intrinsic properties. Specifically, the surface energy for the side facets is about three times smaller than that of the end facets of a wurtzite CdSe quantum rods provided that those end facets are unpassivated by surfactants. ${ }^{52}$ It should be noted that the end facets are polar since they have alternating layers of cations and anions, whereas for the side facets, they are nonpolar due to the equivalent surface cations and anions. ${ }^{51,52}$ Therefore, the passivation of the surface Cd atoms with phosphonic acids reduces the surface energy of the side facets, making them to possess a lower energy than the unpassivated polar (0001) and (0001) facets. Regarding the polar end facets, they are usually not imperfectly passivated by surfactant organic ligands and have higher surface energy, which are the dominant facets responsible for the anisotropic growth of the anisotropic wurtzite nanocrystals. A precise control of the aspect ratios, which is difficult for bulk hexagonal crystals, can be readily achieved in colloidal hexagonal CdSe quantum rods with the aid of organic surfactants. ${ }^{15}$

\section{Synthetic strategies for colloidal semiconductor quantum rods}

\subsection{Kinetically controlled dot/rod in the rod seeded growth method}

The discovery of anisotropic semiconductor CdSe quantum rods was a big surprise because prior to this pioneering work, ${ }^{15}$ colloidal semiconductor nanocrystals were mainly expected to be spherical nanoparticles. Alivisatos and his colleagues ${ }^{15,16}$ demonstrated that the shape of the produced nanoparticles could be successfully achieved by a combination of the growth kinetics of wurtzite CdSe nanocrystals and the organic surfactants. Spherical nanoparticles were obtained if the overall growth rate was low. Anisotropic growth of CdSe nanocrystals with wurtzite structure was highly kinetically driven at an extremely high monomer concentration. The growth process of CdSe quantum rods mainly involves two stages: nucleation and anisotropic growth. Firstly, the nucleation of the reacting precursors produced spherical CdSe spherical nanoparticles, and subsequently the reacting monomer selectively attached much faster on some specific facets of the proceeding nucleation centres than the other facets, leading to elongated CdSe quantum rods.

The invention of the seeded growth approach is inspired by the different growth stages of the above-mentioned anisotropic growth of CdSe quantum rods. ${ }^{17-20}$ Fig. 3a shows the schematic illustration of the anisotropic growth of CdS quantum rods using the CdSe nanocrystals as the seeds. In this approach,
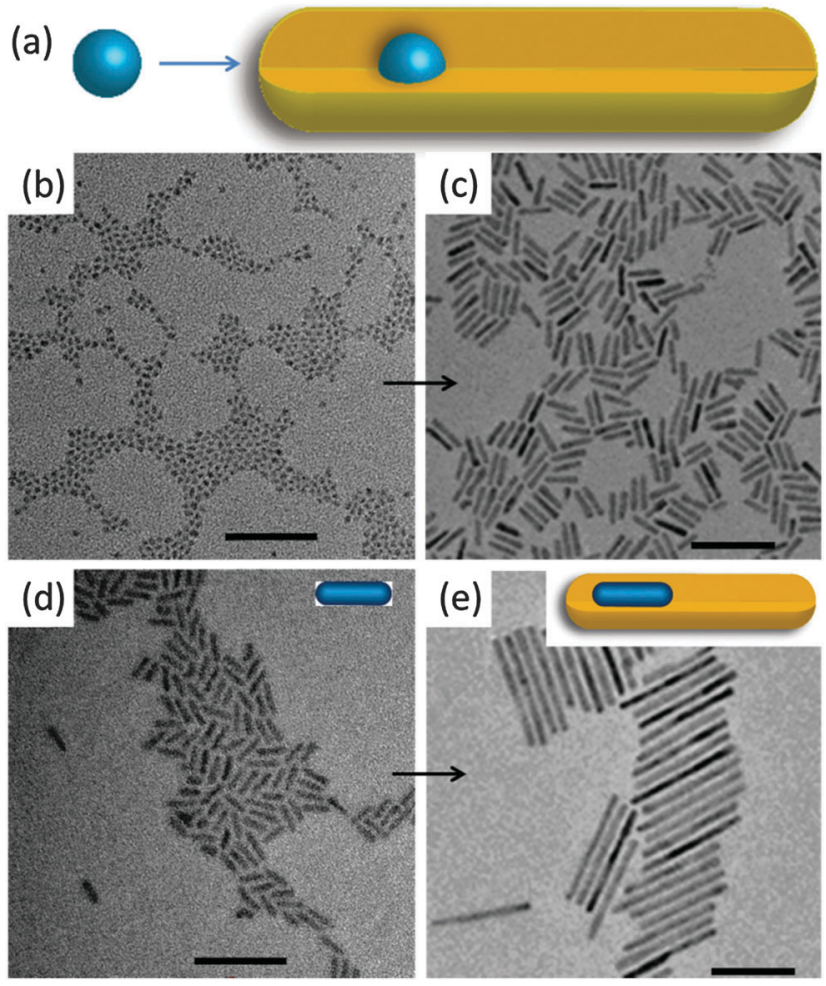

Fig. 3 Seeded growth of colloidal semiconductor quantum rods. (a) Schematic illustration of quantum rod growth by a spherical dot seeded growth approach. TEM images of (b) spherical CdSe quantum dots. (c) CdS quantum rods grown by a seeded growth approach using the spherical quantum dots in (b). (d) Elongated small CdSe quantum rods. (e) CdS quantum rods grown by a seeded growth approach using the small quantum rods in (d). Insets in (d) and (e) show schematic structures of quantum rods. All scale bars are $50 \mathrm{~nm}$. Panels (a)-(c) and (d) and (e) reproduced from ref. 18 and 20, respectively, with permission of the American Chemical Society.

spherical CdSe nanoparticles were firstly synthesized and isolated from the reacting solution for further reactions. In the second step of the synthesis, a certain amount of spherical CdSe nanoparticles were used as the seeds (or nucleation centres). Together with a sulphur precursor which was predissolved in a solution, the seeds were quickly injected into the reaction solution at a high reaction temperature. In the presence of the seeds, which were acting as the nucleation centres, reacting monomers grew on these seeds rather than nucleating themselves since the self-nucleation of the reacting monomers required much more energy. Recently, this seeded growth approach has been modified by Banin and his colleagues. $^{20}$ They proposed a rod in rod seeded growth approach. This synthetic approach is exactly the same as the abovementioned one except that thin CdSe quantum rods, rather than spherical nanoparticles, have been used as the seeds (Fig. 3d and e). The CdSe/CdS quantum rods synthesized using this approach show highly polarized emission, being equal to or up to 1.5 times higher than the polarization of the equivalent spheres in the rod systems.

The merit of this seeded growth approach is that the amount of the nucleation centres can be easily controlled. The high 
reproducibility and feasibility of this seeded growth approach produced CdS quantum rods with high monodispersity and controlled aspect ratios, making it to be one of the most powerful synthetic methods for colloidal semiconductor quantum rods. The availability of highly monodisperse CdS quantum rods with controlled aspect ratios already led to lots of stimulating studies, such as hetero-composites, noble metal-CdS quantum rods hybrid structures with improved photocatalytic and electronic properties, CdS quantum rod superlattices and superparticles with increased photoluminescence anisotropy. ${ }^{8-14,53-70}$

\subsection{Thermodynamic controlled growth by a ripening process}

Current investigations on colloidal semiconductor quantum rods are mainly limited to cadmium chalcogenides primarily due to their facile synthetic accessibility as was demonstrated by the seeded growth approach. ${ }^{1,18-20}$ However, this seeded growth approach, which has proved to be highly reproducible and powerful for the synthesis of cadmium chalcogenide quantum rods, does not work for the synthesis of zinc chalcogenide quantum rods since it produced zinc chalcogenide nanocrystals with either poor quality or uncontrollable aspect ratios. ${ }^{71-87}$ These examples clearly demonstrate that there is a lack of understanding of the factors that influence the growth of colloidal zinc chalcogenide quantum rods. This situation had not improved until recently Jia and his colleagues demonstrated that monodisperse zinc chalcogenide quantum rods with controlled aspect ratios could be successfully synthesized by a ripening process through thermodynamically driven material diffusion. ${ }^{35}$

The mechanism of self-ripening of an individual nanoparticle was firstly proposed by Peng to explain the evolution of elongated CdSe nanocrystals at different growth stages of a growth procedure (Fig. 4a) ${ }^{88,89}$ It states that the transition of the morphology of an individual nanoparticle from one stage to another is significantly dependent on the reacting monomer concentration. In this scheme, 1-dimensionally anisotropic growth is favoured at extremely high monomer concentration. As the chemical reaction proceeds, the growth of the nanoparticles depletes a large amount of the monomers in the reaction solution and largely reduces the concentration of the reacting monomers, which leads to the 3-dimensional growth of the nanocrystals. Further reducing the monomer concentration below a level that is required for the elongated shape growth initiates the 1D/2D-ripening process for the pre-formed elongated nanocrystals, producing thick and short quantum rods, and even dot-shaped nanoparticles if the reaction time was sufficiently long. The ripening mechanism addressed above was employed only to explain the shape evolution of CdSe nanocrystals during the synthetic process and the post-synthesis annealing stage. No such work has been reported in which the ripening mechanism is used for the synthesis of anisotropic nanocrystals until the recent demonstration on the strategy for synthesizing colloidal zinc chalcogenide quantum rods using a thermodynamically controlled approach.

The synthetic protocol for synthesizing zinc chalcogenide quantum rods is illustrated in the flowchart in Fig. 4b. This strategy starts with the synthesis of ultrathin nanowires. In the
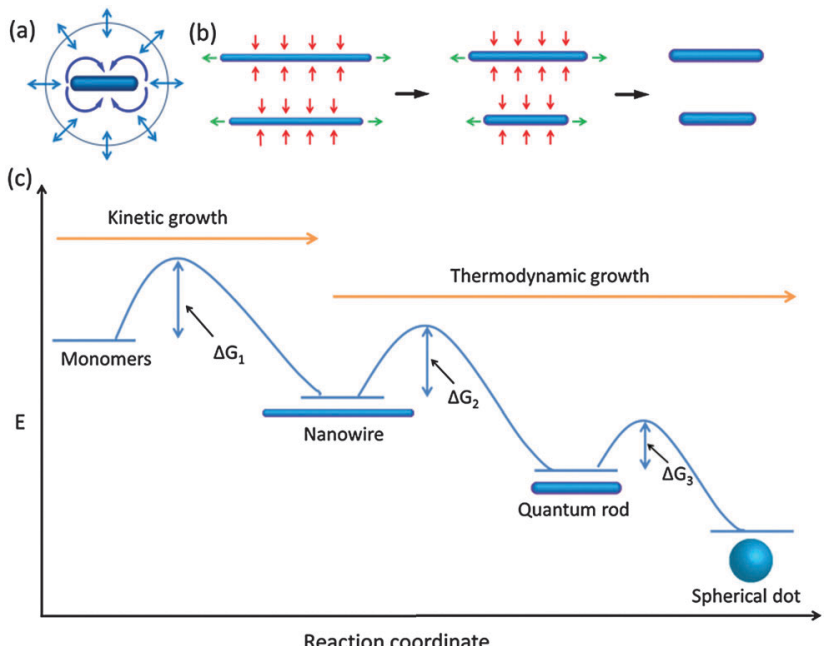

Fig. 4 Crystal growth and evolution of elongated nanoparticles by a ripening process. (a) Schematic illustration of 1D/2D ripening. (b) Mechanism associated with the evolution from nanowires to quantum rods via ripening. (c) Schemes of thermodynamic and kinetic growth of elongated nanocrystals. At high monomer concentrations, the formation of nanowires is preferred whereas at low monomer concentrations, evolution from nanowires to quantum rods and spherical dots is favoured. $\Delta G$ is the activation energy, which can be bridged by the increase of the temperature of the reaction system. Panels (a) and (b and c) reproduced with permission from ref. 88 and 35 , respectively, with permission of the American Chemical Society.

2nd step, at elevated temperature, the material of nanowires diffuses from the end facets to the solution and then from the solution to the side facets, forming quantum rods via a ripening process. Compared with the growth of the initial elongated nanoparticles formed through the chemical reaction of monomers, which is highly kinetically controlled, the growth of the thick and short quantum rods as well as the spherical quantum dots formed from the proceeding elongated nanoparticles is highly thermodynamically controlled (Fig. 4c). It is evident that barriers occur between two consecutive stages associated with the growth and evolution of the nanocrystals. These barriers correspond to the activation energies $(\Delta G)$, which can be bridged by the thermal energy of the reaction system. Therefore, a reasonably high temperature is needed to trigger the ripening process of nanoparticles at a relatively low concentration of the reacting monomers.

As illustrated in Fig. 5a-c, monodisperse ZnSe quantum rods with controlled aspect ratios from their nanowire counterparts have been successfully synthesized use a ripening process via thermodynamically driven material diffusion at an elevated temperature, ca. $280{ }^{\circ} \mathrm{C}$. The associated absorption spectra manifest significant red shifts, which are consistent with the increase of the diameters of the elongated nanoparticles. In addition (Fig. 5d), the sharp features of the (002) plane of the XRD pattern of ZnSe quantum rods corroborate the orientation of the long axis of the wurtzite structure (Fig. 5e). An interesting question is that does this ripening process finally lead to the formation of spherical $\mathrm{ZnSe}$ nanocrystals if the reaction time is sufficiently long? In principle, the transition from elongated 

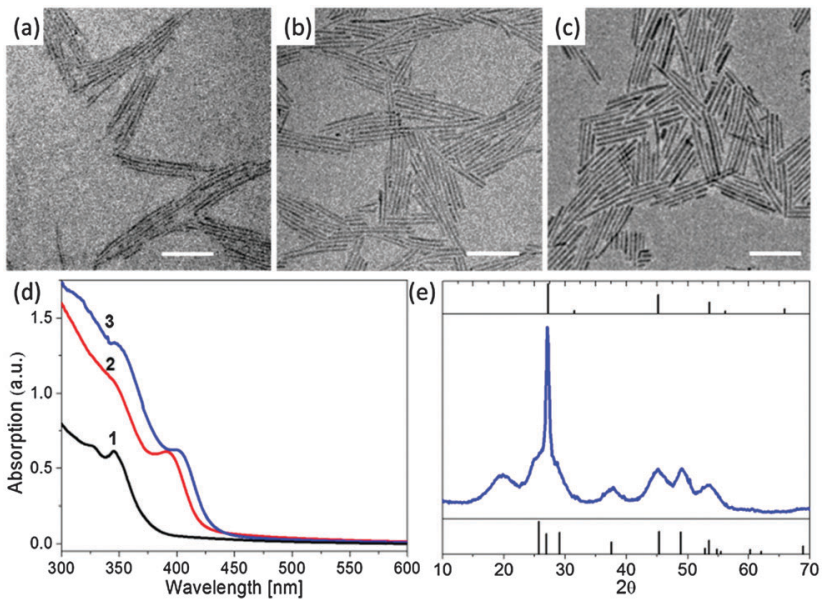

Fig. 5 Evolution of ZnSe quantum rods from ZnSe nanowires. TEM images of the original ZnSe nanowires (a) and the ZnSe quantum rods evolved from the nanowires after $5 \mathrm{~min}$ (b) and $15 \mathrm{~min}$ (c) at $280^{\circ} \mathrm{C}$. All scale bars are $50 \mathrm{~nm}$. (d) Absorption spectra of ZnSe nanocrystals. Curves 1-3 correspond to the materials shown in $(a-c)$, respectively. (e) The XRD pattern of the sample shown in (c). Panels (a)-(e) reproduced with permission from ref. 35 with permission of the American Chemical Society.

quantum rods to spherical nanocrystals is favoured since the system of the spherical nanocrystals has lowest energy within such a thermodynamically controlled growth process. However, we found that after the ZnSe quantum rods reach an equilibrium length, further reaction at high temperature eventually led to the dissolution and decomposition of the obtained quantum rods. This means the synthetic conditions that are needed for the transition from long ZnSe quantum rods to short ones are not necessarily suitable for the transition from short ZnSe quantum rods to their spherical counterparts. Therefore further optimization of the synthetic conditions is required to ensure this transition.

This synthetic strategy has been successfully expanded to other semiconductor materials to construct other quantum rods including $\mathrm{ZnS}$ and $\mathrm{ZnTe}$, demonstrating the generality of this synthetic approach. ${ }^{35} \mathrm{We}$ anticipate that this strategy could also be applied to other colloidal systems, such as noble metals, oxides and insulators, to construct a variety of colloidal 1-D nanocrystals with controlled aspect ratios.

\subsection{Oriented attachment}

The mechanism of oriented attachment was firstly proposed by Banfield and her colleagues to explain the small mis-orientation at the interfaces involved in the particle growth. ${ }^{90,91}$ The concept of oriented attachment is mainly based on the interactions that hold the assembled components together. Those interactions between particles, ranging from electrostatic forces to van de Waals, act as the driving forces for the process of oriented attachment. In recent years, this mechanism has been significantly expanded and used to construct a variety of novel nanostructures with controlled properties, such as luminescent CdTe wires, ${ }^{92} \mathrm{ZnO}$ quantum rods, ${ }^{21} \mathrm{CdTe}$ sheets, ${ }^{93} \mathrm{PbSe}$ sheets ${ }^{94} \mathrm{CdTe}$ ribbons, ${ }^{95} \mathrm{PbSe}$ honeycombs ${ }^{96}$ and ZnSe quantum rod couples. ${ }^{9}$

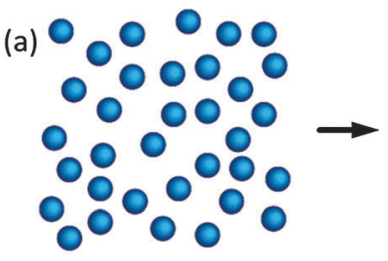

(b)
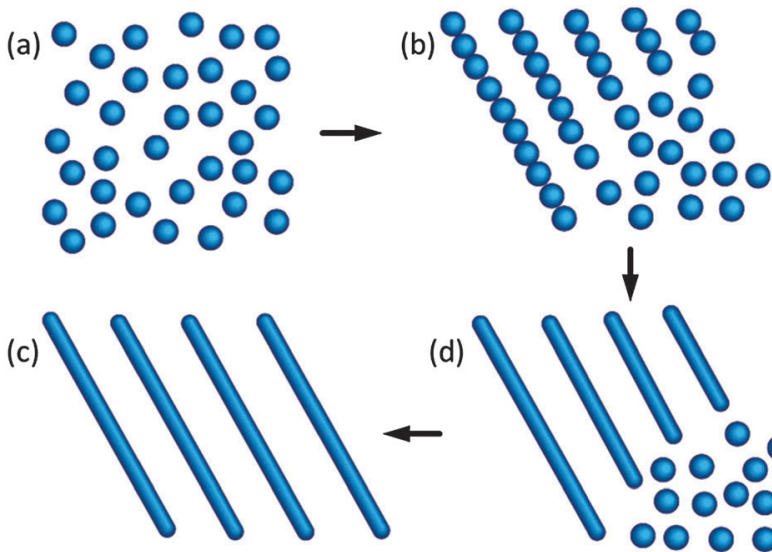

(d)

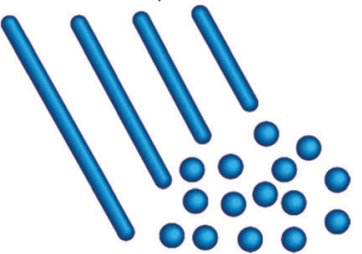

Fig. 6 Schematic illustration of quantum rods formed from isolated spherical dots by an oriented attachment mechanism. Panels (a) $-(d)$ reproduced from ref. 23, with permission of American Chemical Society.

The oriented attachment mechanism can be readily employed to prepare elongated quantum rods with controlled aspect ratios, as illustrated in the scheme in Fig. 6. In this mechanism, isolated individual nanoparticles formed firstly at the early growth stage (Fig. 6a), then specific facets of nanoparticles attached via weak interactions (Fig. 6b), followed by formation of a covalent bond between adjacent facets of nanoparticles, leading to elongated quantum rods (Fig. 6c and d). It is important to mention that, in some instances, partial removal of the surfactants from the surface of the nanoparticles or ligand exchange of the surface passivating molecules from strong ligands to weak ones, which makes some specific facets of nanoparticles to be more reactive, is crucial for the oriented attachment and may trigger the oriented attachment process.

The synthesis of colloidal semiconductor quantum rods based on the oriented attachment mechanism has been demonstrated in a large variety of systems. For example, Weller et al. reported the formation of single crystalline wurtzite $\mathrm{ZnO}$ quantum rods based on the oriented attachment of preformed quasi-spherical $\mathrm{ZnO}$ nanoparticles (Fig. 7a and b). ${ }^{21}$ The intermediate products of the formation of $\mathrm{ZnO}$ quantum rods have different morphologies such as partially fused dimers and oligomers. The width of the obtained $\mathrm{ZnO}$ quantum rods at the early stage of growth $(2 \mathrm{~h}$ reflex) is identical to the diameter of the originally formed $\mathrm{ZnO}$ nanoparticles. The final $\mathrm{ZnO}$ quantum rods show anisotropic wurtzite structure with the crystallographic $c$-axis being elongated. However, the formation of quantum rods based on the oriented attachment mechanism does not necessarily require the starting nanocrystals to be crystallographically anisotropic. Hyeon and his colleagues demonstrated that short quasi-spherical and short rodshaped cubic ZnS nanoparticles, which are crystallographically isotropic, could transform into cubic $\mathrm{ZnS}$ quantum rods by oriented attachment (Fig. $7 \mathrm{c}$ and d). ${ }^{23}$ They also found that aging and annealing of the pre-formed nanoparticles in a weak ligand such as the oleylamine solution at elevated temperatures could increase the yield of the cubic $\mathrm{ZnS}$ quantum rods. The oriented attached mechanism can also be applied to the PbSe system to synthesize PbSe quantum rods, as shown in Fig. 7e and f. ${ }^{24}$ 

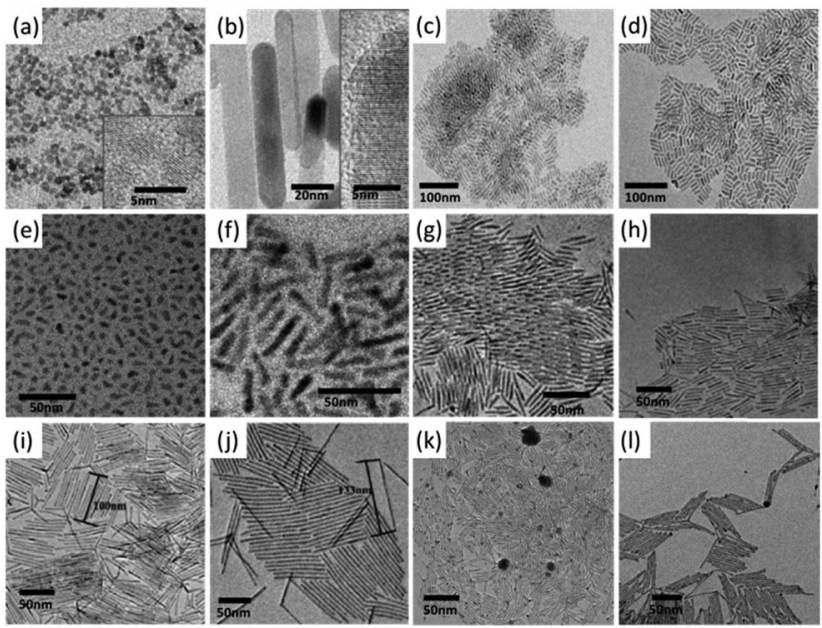

Fig. 7 Semiconductor quantum rods synthesized by the oriented attachment mechanism. TEM images of (a) original spherical ZnO nanoparticles. (b) $\mathrm{ZnO}$ quantum rods formed by the nanoparticles in (a). Insets show high resolution TEM images of the corresponding nanoparticles. (c) Original ZnS quantum rods, (d) ZnS quantum rods formed by the nanoparticles in (c). (e) Original spherical and short rod-shaped PbSe nanoparticles. (f) PbSe quantum rods formed by the nanoparticles in (e). (g) Original CdS quantum rods. (h)-(j) Long CdS quantum rods formed by the nanoparticles in (g). (k) Original $\mathrm{Ag}_{2} \mathrm{~S}$ nanorods, (l) long $\mathrm{Ag}_{2} \mathrm{~S}$ quantum rods formed by the nanoparticles in (k). Panels (a) and (b) reproduced from ref. 21 with permission of Wiley- $\mathrm{VCH}$. Panels (c) and (d) reproduced with permission from ref. 23, panels (e) and (f) reproduced with permission from ref. 24, panels $(\mathrm{g})-(\mathrm{l})$ reproduced with permission from ref. 97 , with permission of the American Chemical Society.

Ryan et al. observed that $\mathrm{CdS}$ and $\mathrm{Ag}_{2} \mathrm{~S}$ quantum rods with controlled aspect ratios could be spontaneously formed at room temperature from their short quantum rod counterparts by oriented attachment (Fig. 7). ${ }^{97}$ The formation of long CdS quantum rods started with the CdS quantum rods with a length of $34 \mathrm{~nm}$ (Fig. $7 \mathrm{~g}$ ). Considering that the facets of the presynthesized CdS quantum rods were tightly bound with the strong phosphonic acid ligands such as HPA and ODPA, alkylamine ligands were employed to wash CdS quantum rods in order to partially substitute surface phosphonic acid ligands passivated on the surface of the CdS quantum rods. Interestingly, amine washing of the preformed CdS quantum rods led to spontaneous end facet attachment of the CdS quantum rods, producing elongated CdS quantum rods with lengths of twice, 3 times and up to four times that of the starting CdS quantum rods (Fig. $7 \mathrm{~h}-\mathrm{j}$ ). It was proposed that the steric effect was responsible for the length limitation of CdS quantum rods that formed by the oriented attachment process. This synthetic approach was also successfully expended to the $\mathrm{Ag}_{2} \mathrm{~S}$ quantum rods, in which long $\mathrm{Ag}_{2} \mathrm{~S}$ quantum rods were produced from the short one via oriented attachment, as shown in Fig. $7 \mathrm{k}$ and $\mathrm{l}$.

\subsection{Catalyst-assisted growth}

The solution-liquid-solid growth mechanism, ${ }^{98,99}$ analogous to the vapour-liquid-solid growth mechanism, ${ }^{100}$ was firstly used in the growth of insoluble whiskers of III-V semiconductors. In this mechanism, a liquid metal or alloy droplet attached to (a)
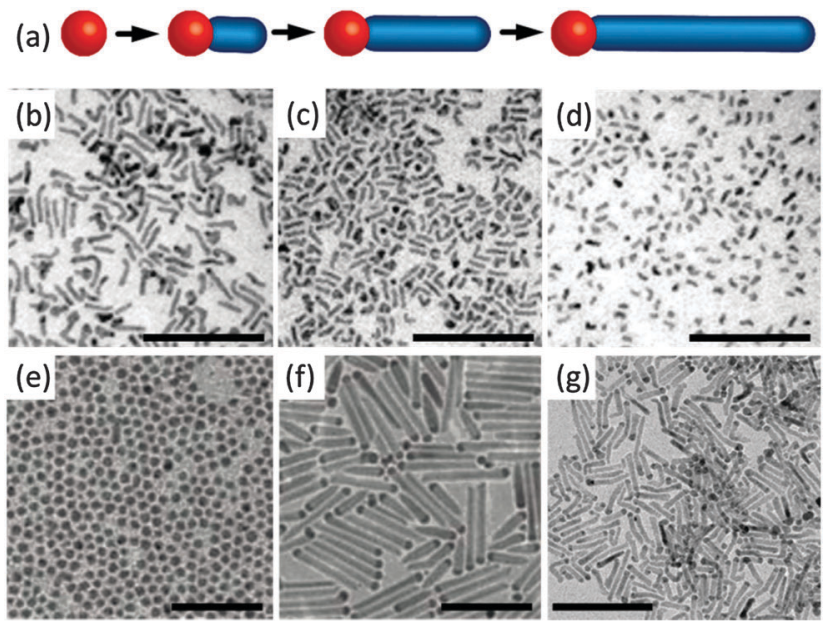

Fig. 8 Catalyst-assisted growth of quantum rods. (a) Schematic illustration of catalyst-assisted growth of quantum rods. TEM images of InAs quantum rods synthesized using $\mathrm{Au}$ as the catalyst with different dimensions (length $\times$ diameter) (b) $22.7 \times 4.0 \mathrm{~nm}$; (c) $15.1 \times 4.1 \mathrm{~nm}$; (d) $9.4 \times$ $4.1 \mathrm{~nm}$. TEM images of (e) Ag spherical nanoparticles with a diameter of $9 \mathrm{~nm}$. (f) ZnS quantum rods synthesized using Ag nanoparticles in (e) as the catalysts; (g) ZnS quantum rods synthesized using $\mathrm{Ag}_{2} \mathrm{~S}$ nanoparticles as the catalyst. Panel (a) reproduced from ref. 98 with permission of AAAS. Panels (b)-(d) reproduced from ref. 28 with permission of McMillan Magazines. Panels (e) and (f) reproduced from ref. 30 with permission of the Royal Society of Chemistry. Panel $(\mathrm{g})$ reproduced with permission from ref. 29 with permission of Wiley-VCH.

the apex of the whisker forms a liquid-solid interface whereas there is a second interface between the droplet and the solution. Reacting monomers in the solution phase are consumed by decomposition at the solution-liquid interface. This is followed by the diffusion of the constituent elements in the droplet, leading to supersaturation that is required for fast whisker growth at the liquid-solid interface (Fig. 8a).

The solution-liquid-solid growth mechanism has been significantly expended for the growth of a variety of soluble semiconductor quantum rods including InAs, ${ }^{28} \mathrm{ZnS},{ }^{29,30}$ and CdSe, ${ }^{31}$ which are expected to be a synthetic challenge for the conventional synthetic approaches. Banin and his colleagues used gold nanocrystals as the catalyst to synthesize cubic InAs quantum rods with controlled lengths and diameters (Fig. $8 \mathrm{~b}-\mathrm{d}$ ). ${ }^{28}$ This work firstly demonstrated that soluble elongated cubic quantum rods could be synthesized by the combination of the solution-liquid-solid growth mechanism by a colloidal chemical route. ${ }^{28}$ Similar studies were conducted by Li et al., in which monodisperse wurtzite $\mathrm{ZnS}$ quantum rods were synthesized using silver nanocrystals as the catalysts (Fig. 8e and f). ${ }^{30}$ Interestingly, the liquid-solid interface of $\mathrm{Ag}-\mathrm{ZnS}$ quantum rods consists of the (200) plane of the $\mathrm{Ag}$ nanocrystal and the (101) plane of ZnS quantum rods. ZnS quantum rods are elongated along the (101) plane rather than the (002) plane (crystallographic $c$-axis of wurtzite structure). Recently, a facile synthetic approach was developed by Wang et al., in which $\mathrm{Ag}_{2} \mathrm{~S}-\mathrm{ZnS}$ quantum rods were synthesized by the thermal decomposition of two single-source precursors using a one-pot method. ${ }^{29}$ The orientation of the long axis of 
ZnS quantum rods is perpendicular to the $c$-axis of the wurtzite crystal, being different from the previously reported $\mathrm{ZnS}$ quantum rods synthesized via the solution-liquid-solid growth mechanism using silver nanocrystals as the catalysts. ${ }^{30}$

The catalyst-assisted growth approach produced metalsemiconductor heterostructures that are highly efficient for charge carrier separation. Therefore this type of heterostructures find wide application in photocatalysiss. ${ }^{63,101,102}$ A main concern with the colloidal semiconductor quantum rods synthesized by the solution-liquid-solid method is associated with the difficulty in removing the metal particles from the semiconductor quantum rods. Obviously, selective chemical etching could be a solution to solve this problem. However, it may also lead to partial dissolution of the semiconductor quantum rods and modification of their surface properties, which therefore profoundly degrades the quality of the colloidal semiconductor quantum rods.

\section{Conclusions and outlooks}

The past two decades witnessed the booming growth of research in colloidal semiconductor quantum rods, which is driven by the consecutive advances in synthesis, properties and applications associated with these materials. Currently existing synthetic strategies for colloidal semiconductor quantum rods already demonstrated their great capabilities to precisely control the length, diameter, aspect ratio and composition of these nanocrystals and this will definitely be significantly advanced by the emerging strategies. It is worth mentioning that there is no such specific synthetic approach that is versatile for the synthesis of all types of semiconductor quantum rods. This means a certain synthetic approach suitable for one semiconductor material is not necessarily applicable to other semiconductor materials. This lies in the fact of the lacking of understanding of the growth habit and parameter windows for the growth of the crystals. Further research efforts need to focus on the combination of intrinsic properties of those semiconductor materials with the control of the synthetic conditions, including the surfactant molecules, monomer concentrations, reaction temperatures, kinetics control and thermodynamics control of the chemical reaction and so on.

The highly toxic heavy metal ions in cadmium and lead compounds have become a main concern since such systems pose a substantial threat to human health and the environment. Because modern society places more severe demands on sustainable and eco-friendly materials, this makes the development of heavy metal-free eco-friendly semiconductor quantum rod alternatives, such as zinc chalcogenide quantum rods and more, ${ }^{35}$ highly desirable. We anticipate fundamental research in those green systems and their emerging application in the fields of photocatalysis, biology and medicine is of interest.

\section{Acknowledgements}

G.J. thanks the Curtin Early Career Research Fellowship for financial support. This work was partially supported by the
Qianjiang Talents Project of Zhejiang Province (Grant No. QJD1402028), the National Natural Science Foundation of China (Grant No. 51472225, 51272243, 21275068 and 21305052) and the International Science \& Technology Cooperation Program of China (Grant No. 2013DFE63070).

\section{Notes and references}

1 J. Hu, L. S. Li, W. Yang, L. Manna, L. W. Wang and A. P. Alivisatos, Science, 2001, 292, 2060.

2 M. Kazes, D. Y. Lewis, Y. Ebenstein, T. Mokari and U. Banin, Adv. Mater., 2002, 14, 317.

3 V. I. Klimov, A. A. Mikhailovsky, S. Xu, A. Malko, J. A. Hollingsworth, C. A. Leatherdale, H. J. Eisler and M. G. Bawendi, Science, 2000, 290, 314.

4 R. Krahne, G. Morello, A. Figuerola, C. George, S. Deka and L. Manna, Phys. Rep., 2011, 501, 75.

5 V. I. Klimov, J. Phys. Chem. B, 2006, 110, 16827.

6 M. Saba, S. Minniberger, F. Quochi, J. Roither, M. Marceddu, A. Gocalinska, M. V. Kovalenko, D. V. Talapin, W. Heiss, A. Mura and G. Bongiovanni, Adv. Mater., 2009, 21, 4942.

7 W. U. Huynh, J. J. Dittmer and A. P. Alivisatos, Science, 2002, 295, 2425.

8 T. Wang, J. Zhuang, J. Lynch, O. Chen, Z. Wang, X. Wang, D. LaMontagne, H. Wu, Z. Wang and Y. C. Cao, Science, 2012, 338, 358.

9 G. H. Jia, A. Sitt, G. B. Hitin, I. Hadar, Y. Bekenstein, Y. Amit, I. Popov and U. Banin, Nat. Mater., 2014, 13, 301.

10 A. Persano, M. De Giorgi, A. Fiore, R. Cingolani, L. Manna, A. Cola and R. Krahne, ACS Nano, 2010, 4, 1646.

11 J. L. Baker, A. Widmer-Cooper, M. F. Toney, P. L. Geissler and A. P. Alivisatos, Nano Lett., 2010, 10, 195.

12 A. Singh, C. Dickinson and K. M. Ryan, ACS Nano, 2012, 6, 3339.

13 J. Zhuang, A. D. Shaller, J. Lynch, H. Wu, O. Chen, A. D. Q. Li and Y. C. Cao, J. Am. Chem. Soc., 2009, 131, 6084.

14 A. Singh, N. J. English and K. M. Ryan, J. Phys. Chem. B, 2013, 117, 1608.

15 X. Peng, L. Manna, W. Yang, J. Wickham, E. Scher, A. Kadavanich and A. P. Alivisatos, Nature, 2000, 404, 59.

16 L. Manna, E. C. Scher and A. P. Alivisatos, J. Am. Chem. Soc., 2000, 122, 12700.

17 Y. Yin and A. P. Alivisatos, Nature, 2005, 437, 664.

18 L. Carbone, C. Nobile, M. D. Giorgi, F. D. Sala, G. Morello, P. Pompa, M. Hytch, E. Snoeck, A. F. Fiore, I. R. Franchini, M. Nadasan, A. F. Silvestre, L. Chiodo, S. Kudera, R. Cingolani, R. Krahne and L. Manna, Nano Lett., 2007, 7, 2942.

19 D. V. Talapin, J. H. Nelson, E. V. Shevchenko, S. Aloni, B. Sadtler and A. P. Alivisatos, Nano Lett., 2007, 7, 2951.

20 A. Sitt, A. Salant, G. Menagen and U. Banin, Nano Lett., 2011, 11, 2054.

21 C. Pacholski, A. Kornowski and H. Weller, Angew. Chem., Int. Ed., 2002, 41, 1188.

22 K. S. Cho, D. V. Talapin, W. Gaschler and C. B. Murray, J. Am. Chem. Soc., 2005, 127, 7140. 
23 J. H. Yu, J. Joo, H. M. Park, S. I. Baik, Y. W. Kim, S. C. Kim and T. Hyeon, J. Am. Chem. Soc., 2005, 127, 5662.

24 W. K. Koh, A. C. Bartnik, F. W. Wise and C. B. Murray, J. Am. Chem. Soc., 2010, 132, 3909.

25 H. Liao, L. Cui, S. Whitelam and H. Zheng, Science, 2012, 336, 1011.

26 D. Li, M. H. Nielson, J. R. I. Lee, C. Frandsen, J. F. Banfield and J. J. De Yoreo, Science, 2012, 336, 1014.

27 W. H. Evers, B. Goris, S. Bals, M. Casavola, J. de Gaaf, R. van Roij, M. Dijkstra and D. Vanmaekelbergh, Nano Lett., 2013, 13, 2317.

28 S. Kan, T. Mokari, E. Rothenberg and U. Banin, Nat. Mater., 2003, 2, 155.

29 S. Shen, Y. Zhang, L. Peng, Y. Du and Q. Wang, Angew. Chem., Int. Ed., 2011, 50, 7115.

30 H. Shen, H. Shang, J. Niu, W. Xu, H. Wang and L. S. Li, Nanoscale, 2012, 4, 6509.

31 L. Ouyang, K. N. Maher, C. L. Yu, J. McCarty and H. Park, J. Am. Chem. Soc., 2007, 129, 133.

32 J. W. Grebinski, K. L. Richter, J. Zhang, T. H. Kosel and M. Kuno, J. Phys. Chem. B, 2004, 108, 9745.

33 F. Shieh, A. E. Saunders and B. A. Korgel, J. Phys. Chem. B, 2005, 109, 8538.

34 J. Sun, L.-W. Wang and W. E. Buhro, J. Am. Chem. Soc., 2008, 130, 7997.

35 G. H. Jia and U. Banin, J. Am. Chem. Soc., 2014, 136, 11121.

36 Y. Xia, P. Yang, Y. Sun, Y. Wu, B. Mayers, B. Gates, Y. Yin, F. Kim and H. Yan, Adv. Mater., 2003, 15, 353.

37 C. Burda, X. Chen, R. Narayanan and M. A. El-Sayed, Chem. Rev., 2005, 105, 1025.

38 Y. W. Jun, J. S. Choi and J. Cheon, Angew. Chem., Int. Ed., 2006, 45, 3414.

39 A. R. Tao, S. Habas and P. Yang, Small, 2008, 4, 310.

40 Z. Lu and Y. Yin, Chem. Soc. Rev., 2012, 41, 6874.

41 D. V. Talapin, J. S. Lee, M. V. Kovalenko and E. V. Shevchenko, Chem. Rev., 2010, 110, 389.

42 V. Lesnyak, N. Gaponik and A. Eychmüller, Chem. Soc. Rev., 2013, 42, 2905.

43 S. Y. Zhang, M. D. Regulacio and M. Y. Han, Chem. Soc. Rev., 2014, 43, 2301.

44 Q. Zhang, S. J. Liu and S. H. Yu, J. Mater. Chem., 2009, 19, 191.

45 K. Liu, N. Zhao and E. Kumacheva, Chem. Soc. Rev., 2011, 40, 656.

46 N. Tian, Z. Y. Zhou, S. G. Sun, Y. Ding and Z. L. Wang, Science, 2007, 316, 732.

47 N. Pradhan, D. Reifsnyder, R. Xie, J. Aldana and X. Peng, J. Am. Chem. Soc., 2007, 129, 9500.

48 C. W. Litton, D. C. Reynolds and T. C. Collins, Zinc Oxide Materials for Electronic and Optoelectronic Device Applications, John Wiley \& Sons, 1st edn, 2011.

49 E. Ohshima, H. Ogino, I. Niikura, K. Maeda, M. Sato, M. Ito and T. Fukuda, J. Cryst. Growth, 2004, 260, 166.

50 L. Manna, E. C. Scher and A. P. Alivisatos, J. Cluster Sci., 2002, 13, 521.

51 J. J. Shiang, A. V. Kadavanich, R. K. Grubbs and A. P. Alivisatos, J. Phys. Chem., 1995, 99, 17417.
52 L. Manna, L. W. Wang, R. Cingolani and A. P. Alivisatos, J. Phys. Chem. B, 2005, 109, 6183.

53 E. Elmalem, A. E. Saunders, R. Costi, A. Salant and U. Banin, Adv. Mater., 2008, 20, 4312.

54 Y. Shemesh, J. E. Macdonald, G. Menagen and U. Banin, Angew. Chem., Int. Ed., 2011, 50, 1185.

55 K. Becker, J. Lupton, J. Müller, A. L. Rogach, D. V. Talapin, H. Weller and J. Feldmann, Nat. Mater., 2006, 5, 777.

56 N. J. Borys, M. J. Walter, J. Huang, D. V. Talapin and J. M. Lupton, Science, 2010, 330, 1371.

57 T. Mokari, E. Rothenberg, I. Popov, R. Costi and U. Banin, Science, 2004, 304, 1787.

58 T. Mokari, C. G. Sztrum, A. Salant, E. Rabani and U. Banin, Nat. Mater., 2005, 4, 855.

59 N. Oh, S. Nam, Y. Zhai, K. Deshpande, P. Trefonas and M. Shim, Nat. Commun., 2014, 5, 3642.

60 Z. Deutsch, L. Neeman and D. Oron, Nat. Nanotechnol., 2013, 8, 649.

61 E. Ploshnik, A. Salant, U. Banin and R. Shenhar, $A d v$. Mater., 2010, 22, 2774.

62 T. Simon, N. Bouchonville, M. J. Berr, A. Vaneski, A. Adrovic, D. Volbers, R. Wyrwich, M. Döblinger, A. S. Susha, A. L. Rogach, F. Jäckel, J. K. Stolarczyk and J. Feldmann, Nat. Mater., 2014, 13, 1013.

63 L. Amirav and A. P. Alivisatos, J. Phys. Chem. Lett., 2010, 1, 1051.

64 K. P. Rice, A. E. Saunders and M. P. Stoykovich, J. Am. Chem. Soc., 2013, 135, 6669.

65 Y. Kelestemur, A. F. Cihan, B. Guzelturk and H. V. Demir, Nanoscale, 2014, 6, 8509.

66 Y. Amit, A. Faust, I. Lieberman, L. Yedidya and U. Banin, Phys. Status Solidi A, 2012, 209, 235.

67 F. Pisanello, G. Leménager, L. Martiradonna, L. Carbone, S. Vezzoli, P. Desfonds, P. D. Cozzoli, J. P. Hermier, E. Giacobino, R. Cingolani, M. De Vittorio and A. Bramati, Adv. Mater., 2013, 25, 1974.

68 H. Schlicke, D. Ghosh, L. K. Fong, H. L. Xin, H. Zheng and A. P. Alivisatos, Angew. Chem., Int. Ed., 2013, 52, 980.

69 M. Zanella, R. Gomes, M. Povia, C. Giannini, Y. Zhang, A. Riskin, M. Van Bael, Z. Hens and L. Manna, Adv. Mater., 2011, 23, 2205.

70 N. Zhao, J. Vickery, G. Guerin, J. I. Park, M. A. Winnik and E. Kumacheva, Angew. Chem., Int. Ed., 2011, 50, 4606.

71 L. S. Li, N. Pradhan, Y. J. Wang and X. Peng, Nano Lett., 2004, 4, 2261.

72 Y. C. Li, X. H. Li, C. H. Yang and Y. F. Li, J. Phys. Chem. B, 2004, 108, 16002.

73 N. Petchsang, L. Shapoval, F. Vietmeyer, Y. Yu, J. H. Hodak, I. M. Tang, T. H. Kosel and M. Kuno, Nanoscale, 2011, 3, 3145 .

74 T. T. Yao, Q. Zhao, Z. Qiao, F. Peng, H. Wang, H. Yu, C. Chi and J. Yang, Chem. - Eur. J., 2011, 17, 8663.

75 P. D. Cozzoli, L. Manna, M. L. Curri, S. Kudera, C. Giannini, M. Striccoli and A. Agostiano, Chem. Mater., 2005, 17, 1296.

76 N. Pradhan and S. Efrima, J. Phys. Chem. B, 2004, 108, 11964. 
77 S. Acharya and S. Efrima, J. Am. Chem. Soc., 2005, 127, 3486.

78 A. B. Panda, S. Acharya and S. Efrima, Adv. Mater., 2005, 17, 2471.

79 J. Zhang, P. C. Chen, G. Shen, J. He, A. Kumbhar, C. Zhou and J. Fang, Angew. Chem., Int. Ed., 2008, 47, 9469.

80 Z. T. Deng, L. Tong, M. Flores, S. Lin, J. X. Cheng, H. Yan and Y. Liu, J. Am. Chem. Soc., 2011, 133, 5389.

81 J. Zhang, K. Sun, A. Kumbhar and J. Fang, J. Phys. Chem. C, 2008, 112, 5454.

82 P. T. K. Chin, J. W. Stouwdam and R. A. J. Janssen, Nano Lett., 2009, 9, 745.

83 Z. Deng, H. Yan and Y. Liu, Angew. Chem., Int. Ed., 2010, 49, 8695.

84 G. Zhu, S. Zhang, Z. Xu, J. Ma and X. Shen, J. Am. Chem. Soc., 2011, 133, 15605.

85 Y. J. Zhang, H. R. Xu and Q. B. Wang, Chem. Commun., 2010, 46, 8941.

86 L. Hou, Q. Zhang, L. Ling, C. X. Li, L. Chen and S. Chen, J. Am. Chem. Soc., 2013, 135, 10618.

87 S. Acharya, S. Sarkar and N. Pradhan, J. Phys. Chem. C, 2013, 117, 6006.

88 Z. A. Peng and X. Peng, J. Am. Chem. Soc., 2001, 123, 1389. 89 X. Peng, Adv. Mater., 2003, 15, 459.

90 R. L. Penn and J. F. Banfield, Science, 1998, 281, 969.

91 J. F. Banfield, S. A. Welch, H. Zhang, T. T. Ebert and R. L. Penn, Science, 2000, 289, 751.
92 Z. Tang, N. A. Kotov and M. Giersig, Science, 2002, 297, 237. 93 Z. Tang, Z. Zhang, Y. Wang, S. C. Glotzer and N. A. Kotov, Science, 2006, 314, 274.

94 C. Scheliehe, B. H. Juarez, M. Pelletier, S. Jander, D. Greshnykh, M. Nagel, A. Meyer, S. Foerster, A. Kornowski, C. Klinke and H. Weller, Science, 2010, 329, 550.

95 S. Srivastava, A. Santos, K. Critchley, K. S. Kim, P. Podsiadlo, K. Sun, J. Lee, C. Xu, G. D. Lilly, S. C. Glotzer and N. A. Kotov, Science, 2010, 327, 1355.

96 M. P. Boneschanscher, W. H. Evers, J. J. Geuchies, T. Altantzis, B. Goris, F. T. Rabouw, S. A. P. van Rossum, H. S. J. van der Zant, L. D. A. Siebbeles, G. Van Tendeloo, I. Swart, J. Hilhorst, A. V. Petukhov, S. Bals and D. Vanmaekelbergh, Science, 2014, 344, 1377.

97 C. O’Sullivan, R. D. Gunning, A. Sanyal, C. A. Barrett, H. Geaney, F. R. Laffir, S. Ahmed and K. M. Ryan, J. Am. Chem. Soc., 2009, 131, 12250.

98 T. J. Trentler, K. M. Hickman, S. C. Goel, A. M. Viano, P. C. Gibbons and W. E. Buhro, Science, 1995, 270, 1791.

99 J. D. Holmes, K. P. Johnston, R. C. Doty and B. A. Korgel, Science, 2000, 287, 1471.

100 R. S. Wagner and W. C. Ellis, Appl. Phys. Lett., 1964, 4, 89.

101 R. Costi, A. E. Saunders and U. Banin, Angew. Chem., Int. Ed., 2010, 49, 4878.

102 R. Costi, A. E. Saunders, E. Elmalem, A. Salant and U. Banin, Nano Lett., 2008, 8, 637. 